Submitted to Appl. Phys. Lett.

\title{
Microwave Near-Field Imaging of Electric Fields in a Superconducting Microstrip Resonator
}

\author{
Ashfaq S. Thanawalla, S. K. Dutta, C. P. Vlahacos, D. E. Steinhauer, \\ B. J. Feenstra, Steven M. Anlage, and F. C. Wellstood \\ Center for Superconductivity Research, Department of Physics, University of Maryland, \\ College Park, Maryland 20742-4111 \\ and \\ Robert B. Hammond \\ Superconductor Technology Incorporated, 460 Ward Drive, Suite F, \\ Santa Barbara, California 93111-2310
}

\begin{abstract}
We describe the use of a cryogenic near-field scanning microwave microscope to image microwave electric fields from superconducting and normal-metal microstrip resonators. The microscope employs an open-ended coaxial probe and operates from 77 to $300 \mathrm{~K}$ in the $0.01-20 \mathrm{GHz}$ frequency range with a spatial resolution of about $200 \mu \mathrm{m}$. We describe the operation of the system and present microwave images of $\mathrm{Cu}$ and $\mathrm{Tl}_{2} \mathrm{Ba}_{2} \mathrm{CaCu}_{2} \mathrm{O}_{8}$ microstrip resonators, showing standing wave patterns at the fundamental and second harmonic frequencies.
\end{abstract}

PACS number: 85.25.Am, 41.21.-q, 74.25.Nf, 07.79.-v 
The demonstration of low microwave loss in high- $\mathrm{T}_{\mathrm{c}}$ superconductors at $77 \mathrm{~K}$ has opened up the possibility for commercial applications which require performance beyond that achievable with normal-metal devices. Unfortunately, present-day high- $\mathrm{T}_{\mathrm{c}}$ microwave circuits have shown performance-limiting problems including non-linearity, intermodulation, and power-dependent characteristics. ${ }^{1}$ One important class of nonlinearities arises from vortex motion at weak links and grain boundaries ${ }^{2}$ and is exacerbated by large current densities found near patterned edges. ${ }^{1}$ Most techniques used to characterize these problems involve global measurements which determine the response of the device as a whole. $^{3-4}$ To obtain a complete microscopic picture of nonlinearity in superconducting rf devices, spatially resolved probes of microwave current distribution, ${ }^{5}$ electric and magnetic field, ${ }^{6}$ loss, ${ }^{7,8}$ and microstructure are needed. Conventional microwave electric field imaging techniques include using modulated perturbations, ${ }^{9}$ electro-optic techniques, ${ }^{10}$ scanning force potentiometry, ${ }^{11}$ and transmission line probes ${ }^{12}$ which locally extract the signal of interest. In this Letter, we describe the imaging of microwave electric fields in superconducting devices using a near-field scanning microwave microscope that operates from 77 to $300 \mathrm{~K}$. We apply this technique to a prototype device, the single-pole microstrip resonant filter, and measure the response of both a normal metal and a superconducting version.

A schematic illustration of our experimental arrangement is shown in Fig. 1. Microwave power is supplied by an HP $83620 \mathrm{~B}$ signal generator with a frequency range from 0.01 to $20.00 \mathrm{GHz}$. The power is amplified by an HP 8349B amplifier to about 100 $\mathrm{mW}$ and fed to the microstrip resonator through an isolator and a capacitive coupler. We use an open-ended coaxial probe to sense the electric fields from the microstrip. The probe is connected through an isolator to a matched $50 \Omega$ diode which rectifies the rf signal to produce a dc output proportional to power. This dc output is amplified, filtered, and recorded by a computer.

The outer conductor of the coaxial probe shields the inner conductor from lateral components of the field, allowing us to measure mainly the normal component of electric field $E_{z}$. The field $E_{z}$ from the microstrip induces a net charge $Q_{I}$ on the center conductor of the probe. ${ }^{13}$ According to Gauss's law, $Q_{I}=\varepsilon_{0} E_{z} A$ where $A \approx 0.03 \mathrm{~mm}^{2}$ is the area of 
the face of the center conductor. The spatial resolution is limited by the diameter of the center conductor of the probe $(200 \mu \mathrm{m})$ or the height of the probe above the device, whichever is greater. ${ }^{14}$ We can find the absolute magnitude of $E_{z}$ by relating $Q_{I}$ to the power $\mathrm{P}$ detected at the diode. The total current induced on the probe face by the electric field is $I=d Q_{I} / d t=i \omega \varepsilon_{0} E_{z} A$, where $\omega$ is the frequency. For a lossless transmission line with a characteristic impedance $\mathrm{Z}_{0}=50 \Omega$, the power detected by the matched diode is $\mathrm{P}$ $=\mathrm{I}^{2} \mathrm{Z}_{0}$. Therefore, one finds $\mathrm{E}_{\mathrm{z}}=\left[\mathrm{P} /\left(\omega^{2} \varepsilon_{\mathrm{o}}^{2} \mathrm{~A}^{2} \mathrm{Z}_{\mathrm{o}}\right)\right]^{1 / 2}$.

The cryogenic part of the setup (see dashed box in Fig.1) is enclosed in a vacuum can which is inserted in a liquid-nitrogen cooled Cryofab Model CSM 6042 Cryostat. By heating the microstrip, images can be obtained at temperatures from 77 to $300 \mathrm{~K}$. To take an image, the microscope is cooled, the probe height is set, and the diode output is recorded as the microstrip is moved in the $\mathrm{x}$ and $\mathrm{y}$ directions using a computer-controlled cryogenic two-axis translation stage.

To investigate the capabilities of our system, we imaged a test copper microstrip resonator. The resonator consists of a $350 \mu \mathrm{m}$ thick circuit board (FR-4 fiberglass) which is coated on both sides with copper. One side serves as a ground plane, while the other is patterned into a microstrip which is $1.1 \mathrm{~mm}$ wide and $\mathrm{L}=7.6 \mathrm{~mm}$ long. The resonator is mounted in a $\mathrm{Cu}$ package and microwave signals can be capacitively coupled in and out of the resonator at one end (see Fig. 2a inset).

To determine the resonant frequency of the microstrip, we positioned the probe at one of the open ends (point $\mathrm{N}$ in Fig. 1) at a height of $1 \mathrm{~mm}$. We then applied $100 \mathrm{~mW}$ of incident power to the capacitive coupler and recorded the diode voltage as a function of frequency. Figure $2 \mathrm{a}$ shows the rf power at the diode for temperatures of 77 and $300 \mathrm{~K}$. Each curve shows a single large resonance, some small secondary resonances, and noise; the small resonances are due to reflections off components and connectors within the system. We estimate the loaded quality factor $\mathrm{Q}$ of the resonator by fitting the overall frequency response with a single Lorentzian lineshape. We find that the $\mathrm{Q}$ of the microstrip increases substantially as the temperature is lowered, mainly due to the improved conductivity of the copper; $\mathrm{Q}=40$ at $300 \mathrm{~K}$, while $\mathrm{Q}=92$ at $77 \mathrm{~K}$. From independent 
broadband transmission measurements at $300 \mathrm{~K}$ we determined the $\mathrm{Q}$ to be within $10 \%$ of the value given above.

At room temperature, the $\mathrm{Cu}$ resonator shows a peak in the detected power at about $9.64 \mathrm{GHz}$, while at $77 \mathrm{~K}$ the peak has shifted to $9.95 \mathrm{GHz}$. Part of the increase in the resonant frequency upon lowering the temperature can be attributed to the decreased inductance from the reduced skin depth $(\sim 10 \mathrm{MHz})$ and thermal contraction of the $\mathrm{Cu}$ microstrip ( 20 MHz). ${ }^{15}$ From independent capacitance measurements, we found that the $310 \mathrm{MHz}$ total shift is mainly due to the dielectric constant of the fiberglass substrate decreasing by $10 \%$ upon cooling.

The 9.5 - $10 \mathrm{GHz}$ resonance in Fig. 2a corresponds to the fundamental standing wave mode for the microstrip. This is demonstrated in Fig. 3a, which shows an image of the magnitude of $\mathrm{E}_{z}$ above the $\mathrm{Cu}$ microstrip resonator at $77 \mathrm{~K}$. During the scan, the frequency of the source was fixed at the resonant frequency $(9.95 \mathrm{GHz})$ and the probe was held about $1 \mathrm{~mm}$ above the microstrip. The image shows a maximum signal at the two open ends of the microstrip conductor (corresponding to the voltage antinodes), and a minimum in the middle (corresponding to the voltage node), as expected.

We next imaged a $\mathrm{Tl}_{2} \mathrm{Ba}_{2} \mathrm{CaCu}_{2} \mathrm{O}_{8}$ (TBCCO) microstrip resonator. ${ }^{16}$ The resonator has a $650 \mathrm{~nm}$ thick film of TBCCO deposited on both sides of a $420 \mu \mathrm{m}$ thick, $4.7 \mathrm{~mm} \mathrm{x}$ $9.9 \mathrm{~mm} \mathrm{MgO}$ substrate. One side serves as a ground plane, while the other side is patterned into a microstrip with a width of $150 \mu \mathrm{m}$ and a length $\mathrm{L}=7.1 \mathrm{~mm}$. We mounted the resonator in a $\mathrm{Cu}$ package and capacitively coupled rf power to one end. Figure $2 \mathrm{~b}$ shows a frequency scan taken at $77 \mathrm{~K}$ (at point $\mathrm{N}$ in Fig. 1), with the probe at a height of about $250 \mu \mathrm{m}$ above the microstrip. Note the much smaller frequency range, as compared to Fig. 2a, indicating the substantially higher $\mathrm{Q}$ of the superconducting resonator.

At $77 \mathrm{~K}$ we find a resonance at about $8.205 \mathrm{GHz}$. The resonance shifts to lower frequencies (see Fig. 2b inset) and diminishes in magnitude as the temperature is raised toward $T_{c} \sim 103 \mathrm{~K}$. No resonance was found above $T_{c}$. The decrease in resonant frequency at higher temperatures is due to the increase in penetration depth $\lambda$ of the superconductor. The temperature dependence of the resonant frequency $\mathrm{f}_{0}$ was fit using a BCS $\mathrm{s}$-wave temperature dependence ${ }^{17}$ for $\lambda(\mathrm{T})$ (solid line in Fig. $2 \mathrm{~b}$ inset). The fit yields $\mathrm{T}_{\mathrm{c}}=$ 
103.2 $\mathrm{K}$ and $\lambda(0)=0.24 \mu \mathrm{m}$. The $\mathrm{T}_{\mathrm{c}}$ is in good agreement with separate ac-susceptibility measurements $(101-103 \mathrm{~K})$, and $\lambda(0)$ is in good agreement with an independent determination by Willemsen et al. $(\lambda(0)=0.26 \mu \mathrm{m})^{18}$ in similar TBCCO thin films. The loaded Q goes from about 1200 at $77 \mathrm{~K}$ to about 480 at $100 \mathrm{~K}$, with a temperature dependence very similar to the one observed for $\mathrm{f}_{0}$. The measured $\mathrm{Q}$ at $77 \mathrm{~K}$ allows us to put an upper bound on the surface resistance of TBCCO: $\mathrm{R}_{\mathrm{s}} \bullet 11.3 \mathrm{~m} \Omega$ at $8.205 \mathrm{GHz}$. However, the $\mathrm{Q}$ is about a factor of 2 lower than the value measured using a network analyzer in the absence of the probe, suggesting that coupling to the probe contributes significant loss.

The lower image in Fig. 3b shows $\left|E_{z}\right|$ above the TBCCO microstrip resonator, measured at $77 \mathrm{~K}$. The frequency of the source is fixed at the resonant frequency $(8.205$ $\mathrm{GHz}$ ) and the probe is $180 \mu \mathrm{m}$ above the microstrip. As with the $\mathrm{Cu}$ resonator, we observe a maximum electric field at the two open ends, as expected for the fundamental mode. However, the field strength is somewhat different at the two antinodes. This is probably due to the presence of the input capacitive coupling pin on the resonator's left hand side, which perturbs the strip's simple geometry and also affects the perturbation of the resonance frequency by the probe. In addition, note that $\mathrm{E}_{\mathrm{z}}$ is much larger than in Fig. $3 \mathrm{a}$, due to the higher $\mathrm{Q}$ of the TBCCO microstrip and the smaller probe-sample separation.

The issue of probe perturbation is relevant to all quantitative electric field measurement techniques $^{9-12}$ and is a topic of ongoing research. In order to evaluate the extent to which perturbations of the resonant frequency affect our images, we also imaged the electric field in a frequency-following mode. In this mode, the frequency of the source is kept on the resonance of the microstrip as the probe is scanned across the resonator. ${ }^{19}$ Clearly this technique will not work at a node, where no signal is present. The upper image in Fig. 3b shows the result for such a scan, measured at the right antinode of the fundamental mode for the TBCCO microstrip at a height of $230 \mu \mathrm{m}$. In comparison to the fixed-frequency image, $\mathrm{E}_{\mathrm{z}}$ shows a different curvature, producing less peaked antinodes. The difference between the two images is due to shifting of the high $\mathrm{Q}$ resonance frequency by the probe, which is not accounted for in the fixed frequency image. On the other hand, neither image shows the $\cos ^{2}(\pi \mathrm{x} / \mathrm{L})$ dependence seen in Fig. 3a. This is 
mainly due to the smaller aspect ratio of the TBCCO microstrip, which tends to concentrate the electric field at the ends.

For comparison, Fig. 3c shows an electric field image obtained at $16.2 \mathrm{GHz}$, where there is also a peak in the frequency response of the microstrip. For this image the probe was held $250 \mu \mathrm{m}$ above the microstrip. We see a complete standing wave pattern with antinodes at the two open ends and the middle of the microstrip, as expected for the second harmonic resonance. The TBCCO strip appears to be fairly well resolved, consistent with a spatial resolution on the order of the $200 \mu \mathrm{m}$ probe size and the probe-sample separation. Finer tips and smaller probe-sample separations can be used to achieve higher spatial resolution.

In conclusion, we have demonstrated a simple broadband microwave imaging system which allows us to measure the normal component of electric field near an operating high frequency device at cryogenic temperatures. The system has achieved about $200 \mu \mathrm{m}$ spatial resolution using only simple microwave instrumentation combined with a cryogenic scanner, establishing the basis for a microscopic examination of superconducting microwave devices.

We wish to gratefully acknowledge R. Newrock for fabrication of the cryogenic scanner and Lucia V. Mercaldo for the penetration depth analysis. This work has been supported by the National Science Foundation NSF grant No. ECS-9632811, NSFMRSEC grant No. DMR-9632521, and by the Maryland Center for Superconductivity Research. 


\section{Figure Captions}

Fig. 1. Schematic illustration of the cryogenic microwave microscope. The dashed box indicates the cryogenic section.

Fig. 2. (a) rf Power at the diode detector vs. frequency for the $\mathrm{Cu}$ microstrip at 77 and 300 K. Inset shows schematic of the microstrip and $\mathrm{Cu}$ package. (b) rf Power at the diode vs. frequency for TBCCO microstrip at 77 and $100 \mathrm{~K}$. The corresponding magnitude of the normal component of electric field at the probe is indicated on the right axes. Inset shows temperature dependence of the resonant frequency $\mathrm{f}_{0}$ along with a fit to BCS-theory (solid line).

Fig. 3. (a) Magnitude of the normal component of electric field $\mathrm{E}_{\mathrm{z}}$ above a $\mathrm{Cu}$ microstrip excited at its fundamental frequency ( $f=9.95 \mathrm{GHz}$ ). (b) $\mathrm{E}_{\mathrm{z}}$ above a TBCCO microstrip excited at its fundamental frequency ( $\mathrm{f}=8.205 \mathrm{GHz}$, lower image). For comparison, the upper image shows $\mathrm{E}_{\mathrm{z}}$ measured using a frequency following technique. (c) $\mathrm{E}_{\mathrm{z}}$ above a TBCCO microstrip excited at its second harmonic frequency $(f=16.2 \mathrm{GHz})$. The contour lines are labeled with field strength in $\mathrm{V} / \mathrm{mm}$. In all cases, power is fed into the microstrip on the left hand side and the temperature is $77 \mathrm{~K}$. 


\section{References}

1. Z.-Y. Shen, High-Temperature Superconducting Microwave circuits, (Artech House, Boston, 1994).

2. J. Halbritter, J. Appl. Phys. 68, 6315 (1990); J. Halbritter, J. Supercond. 8, 691 (1995).

3. D. E. Oates, P. P. Nguyen, G. Dresselhaus, M. S. Dresselhaus, C. W. Lam, and S. M. Ali, J. Supercond. 5, 361 (1992).

4. G. Hampel, B. Batlogg, K. Krishana, N. P. Ong, W. Prusseit, H. Kinder, and A. C. Anderson, Appl. Phys. Lett. 71, 3904 (1997).

5. H. S. Newman and J. C. Culbertson, Microwave Opt. Technol. Lett. 6, 725 (1993).

6. R. C. Black, F. C. Wellstood, E. Dantsker, A. H. Miklich, D. Koelle, F. Ludwig, and J. Clarke, Appl. Phys. Lett. 66, 1267 (1995).

7. G. Hampel, P. Kolodner, P. L. Gammel, P. A. Polakos, E. De Obaldia, P. M. Mankiewich, A. Anderson, R. Slattery, D. Zhang, G. C. Liang, and C. F. Shih, Appl. Phys. Lett. 69, 571 (1996).

8. J. Gallop, L. Hao, and F. Abbas, Physica C. 282-287, 1579 (1997).

9. T. P. Budka, S. D. Waclawik, and G. M. Rebeiz, IEEE Trans. Microwave Theory Tech. 44, 2174 (1996), and references therein.

10. K. J. Weingarten, M. J. W. Rodwell, and D. M. Bloom, IEEE J. Quantum Electron. 24, 198 (1988), and references therein.

11. A. S. Hou, F. Ho, and D. M. Bloom, Electron. Lett. 28, 2302 (1992).

12. D. W. van der Weide and P. Neuzil, J. Vac. Sci. Technol. B 14, 4144 (1996).

13. Note that in the presence of the metallic probe, the magnitude of the field can be significantly different from the case with the probe absent.

14. Steven M. Anlage, C. P. Vlahacos, S. K. Dutta, and F. C. Wellstood, IEEE Trans. Appl. Supercond. 7, 3686 (1997).

15. B. J. Feenstra, C. P. Vlahacos, Ashfaq S. Thanawalla, D. E. Steinhauer, S. K. Dutta, F. C. Wellstood, and Steven M. Anlage, IEEE MTT-S Int. Microwave Symp. Digest, 965 (1998).

16. Superconductor Technology, Inc., sample TM0171 - $R_{1}, R_{2}, R_{3}$. 
17. One expects the BCS temperature dependence to be obeyed for a d-wave superconductor near $\mathrm{T}_{\mathrm{c}}$, in the absence of critical fluctuations.

18. B. A. Willemsen, K. E. Kihlstrom, T. Dahm, D. J. Scalapino, B. Gowe, D. A. Bonn, W. N. Hardy, submitted to Phys. Rev. B.

19. A similar technique is reported in: D. E. Steinhauer, C. P. Vlahacos, S. K. Dutta, F. C. Wellstood, and Steven M. Anlage, Appl. Phys. Lett. 71, 1736 (1997). 


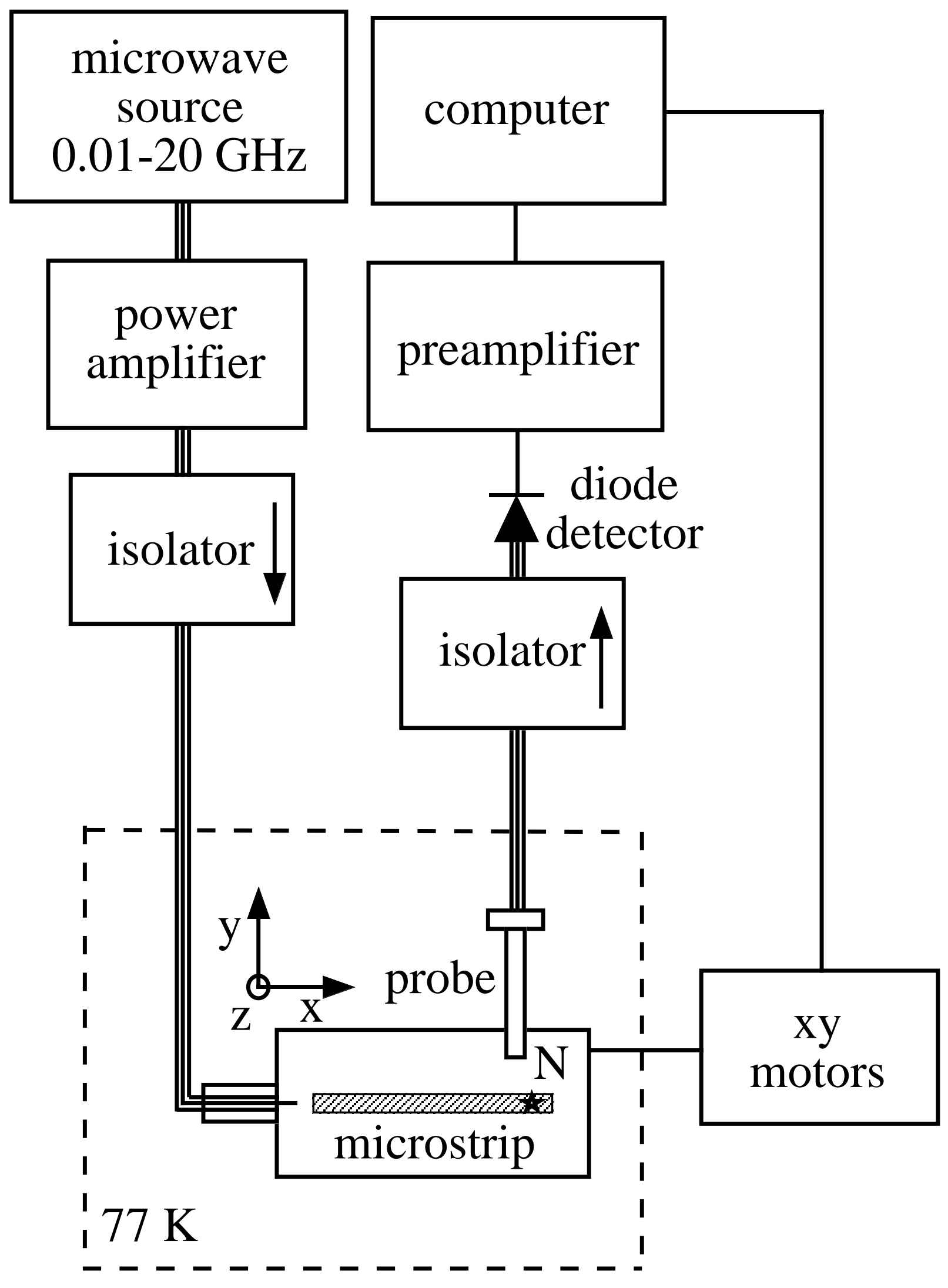



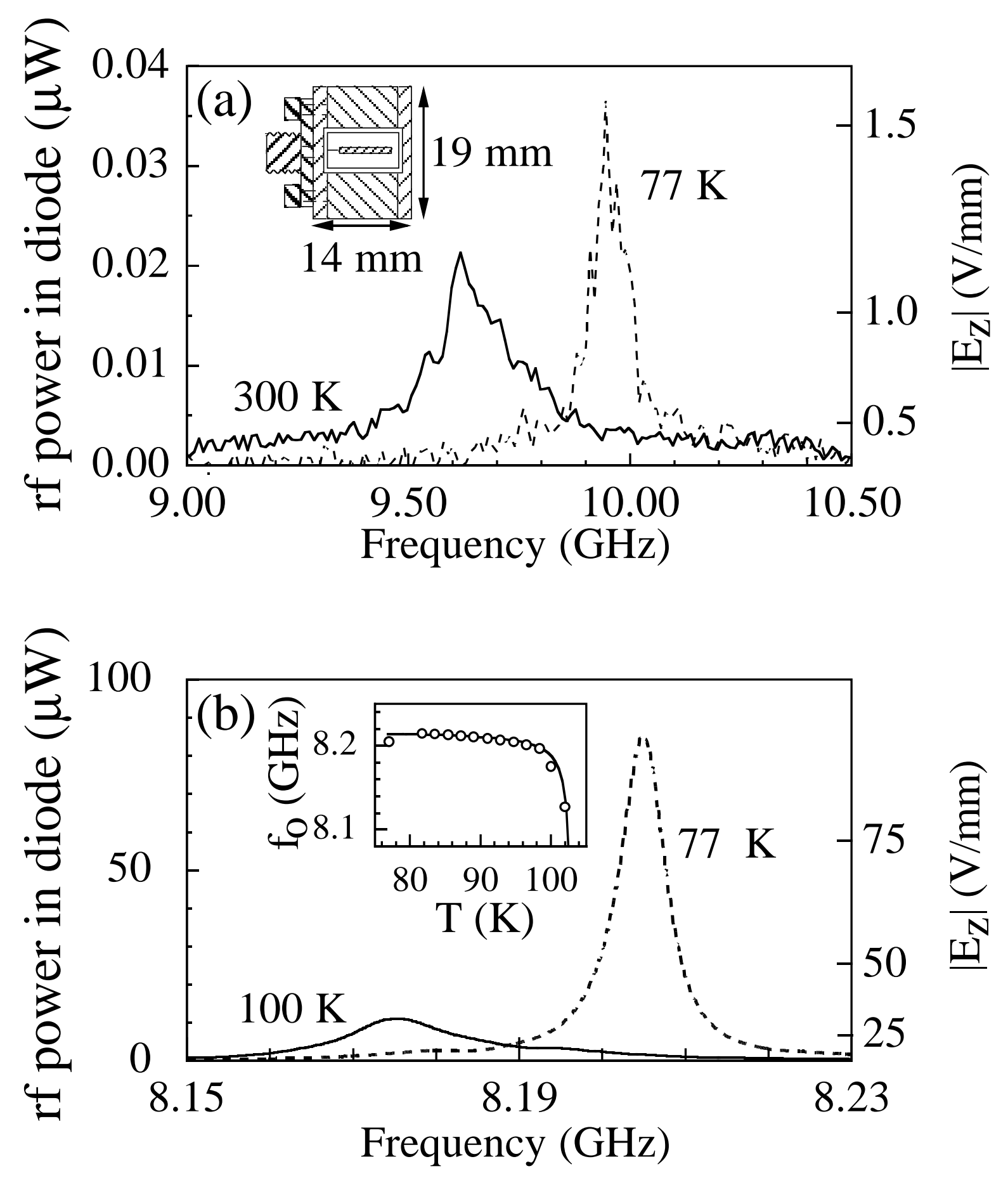
(a)
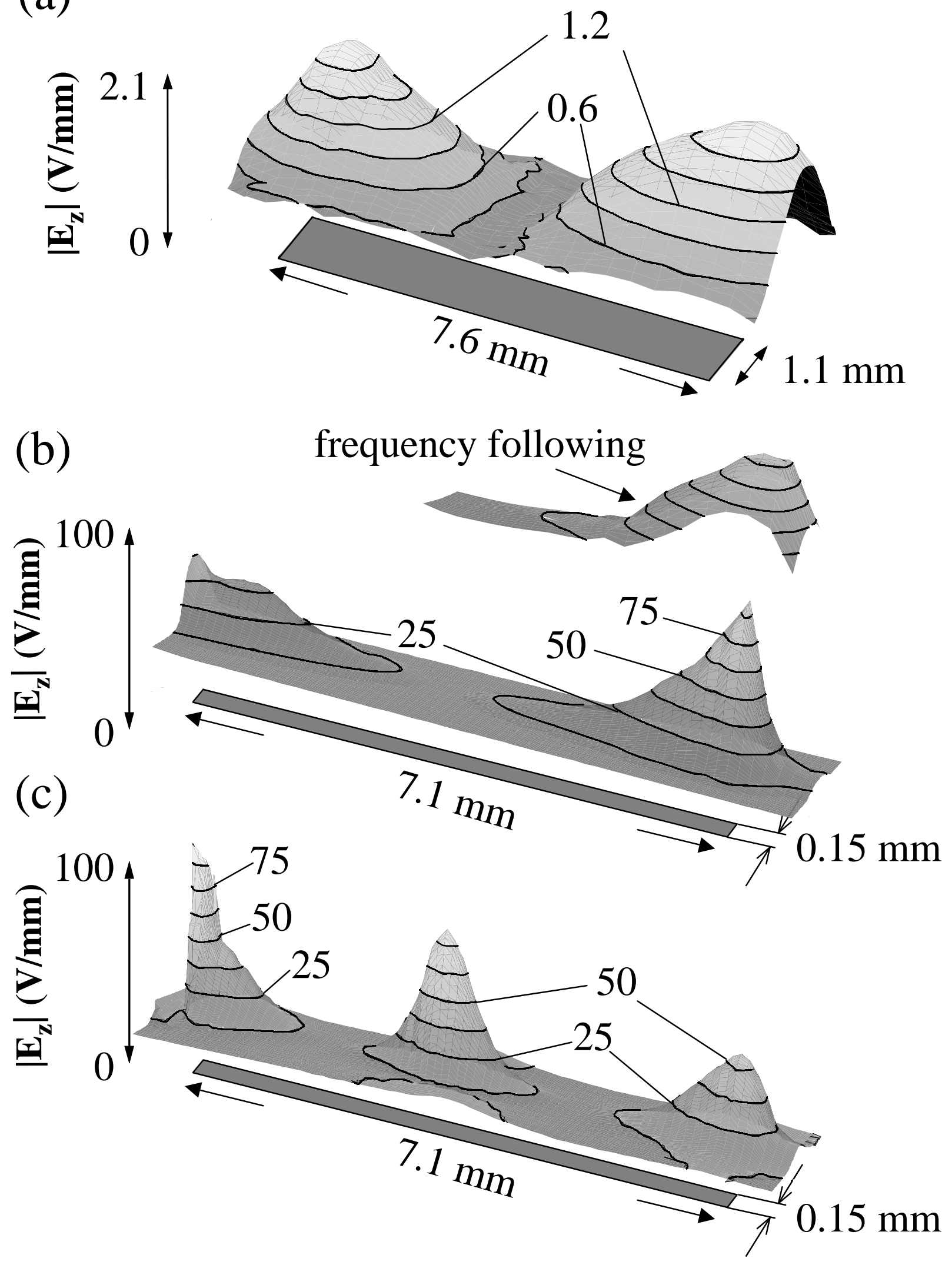

Fig 3, Thanawalla et al. 